\title{
A New Look at Definite Descriptions
}

\author{
Mario Bunge \\ Foundations and Philosophy of Science Unit, McGill University
}

Definite descriptions, such as 'my mother', 'our Lord', 'the square of 2 ', and 'the golden mountain', can pose some subtle logical and semantical problems. Particularly when they purport to describe something that happens not to exist. Some of the problems definite descriptions raise are these:

(i) Logical problems

(a) The problem of supplying one or more adequate analyses of definite descriptions, either in the form of definition (hence trivialization) or in the form of theories. In particular, are Russell's and Hilbert-Bernays' resolutions of definite descriptions into uniqueness and existence adequate?

(b) The problem of ascertaining the conditions under which a definite description can be treated as a name for purposes of inference. In particular, can 'the immortal man' function as a name in a statement occurring in an argument?

(ii) Semantical problems

(a) Finding out whether a statement containing a definite description makes sense in a given context. In particular, does 'the mad hatter' make sense absolutely, i.e. irrespective of context?

(b) Finding out under what conditions a statement containing a definite description has both a referent and a truth value. For example, what is the referent of "the present king of France" and what the truth 
value of "The present king of France is bald"?

This paper addresses itself to these two batches of problems. It starts by questioning the received doctrine.

\section{Inadequacy of Russell's solution}

It took the Russell to realize that definite descriptions pose thorny problems and that they should be analyzed with the help of the then young mathematical logic. (Frege too had seen the problem but he offered no elaborate solution to it.) Russell's now classical "theory", or rather definition, ${ }^{1}$ boils down to this: a definite description presupposes existence and it indicates uniqueness. That is, "the so-and-so is such-and-such" must be analyzed as "There is a unique thing that is so-and-so and such-andsuch". In symbols,

$$
G((2 x) F x)=\mathrm{df}(E x)(F x \& G x \&(y)(F y \rightarrow y=x))
$$

Hilbert and Bernays ${ }^{2}$ give a different version of the idea that "the soand-so" is no more and no less than "there is a so-and-so and it is unique". They posit the rule of inference

$$
\begin{gathered}
(E x) F x \\
(x)(y)(F x \& F y \rightarrow x=y) \\
\hline F\left(\left({ }^{\prime} x\right) F x\right)
\end{gathered}
$$

subject to certain restrictions. The differences between $R$ and $H B$ are these: (a) while $R$ is a definition, $\mathrm{HB}$ is a rule of inference that, if accepted, must be added to the rules of the predicate calculus; (b) $R$ is contextual, in the sense that the referent must be assigned a further property (i.e. $G$ ) in addition to the one it exemplifies uniquely (i.e. $F$ ); on the other hand HB makes no such demand but in compensation it forces us to employ correct yet redundant expressions such as 'my mother is a mother' and 'the square of 2 is a square of 2'; (c) while in $R$ existence and uniqueness are fused together, in HB they are stated separately. 
Each of the above explications, $R$ and $H B$, has its merits and demerits. In particular, $R$ is simpler than $H B$, while $H B$ involves a neat separation of existence from uniqueness. However, this detachment of the two concepts should be carried even further :one should be able to describe nonexisting objects as well as objects the existence of which has not yet been established. Thus the bachelor should be able to say 'My wife does not exist' and the cosmologist should be able to wonder whether there is such thing as the center of the world. Yet neither $R$ nor $H B$ render this possible, as both make existence a condition for definite description. I submit that this makes both $\mathrm{R}$ and HB unsuitable. The same objection holds for other explications of the definite description. ${ }^{3}$

Both in mathematics and in factual science questions of uniqueness are separate from questions of existence: an object satisfying a certain description may not exist or, if it does, it may not be unique. For example, the theory of differential equations contains separate existence theorems and uniqueness theorems. And in theoretical physics one can often give an unambiguous characterization of a certain object the actual existence of which is far from certain: thus one may speculate on the ground state of a transuranian atom that has not yet been and may never be brought into existence.

Whether in mathematics or in factual science, when confronted with validating uniqueness claims one proceeds in this way. One first assumes or investigates uniqueness under the assumption of existence: it would be impossible to look for unique nonexistents. In other words, uniqueness theorems take the form: "If there is an $x$ with the property $F$, then there is no $y$, other than $x$, that exemplifies $F$ ". Thus uniqueness proofs depend on existence proofs but not conversely, and similarly for the empirical validation of hypotheses of factual uniqueness. But this does not mean that 
the concept of uniqueness depends upon the one of existence. In fact these two concepts are not interdefinable. And uniqueness assumptions are not deducible from existence assumptions alone.

Now, I submit that definite descriptions indicate only uniqueness: that they are by themselves uncommitted to existence (even though establishing the latter is necessary for proving the former). Otherwise the bachelor could make no jokes about his wife, the atheist could not argue about the Christian god, the physicist could not speculate about Maxwell demons, and the cosmologist could make no hypotheses about the center of the universe. This being so, we can acept no explication of definite description that involves existence. In this respect then $\mathrm{HB}$ is as inadequate as $\mathrm{R}$. We must therefore look for a different characterization. We shall come up with two definitions, neither of which will force us to augment the set of rules of inference.

\section{An elementary analysis of definite descriptions}

The standard analysis of the definite descritpion equates it with existence and uniqueness. Ours will drop existence and keep uniqueness. Now, the uniqueness condition can be expressed in a number of languages. In the present section we shall propose an analysis of definite descriptions within predicate logic.

We are concerned with uniqueness irrespective of existence, which should be assumed or denied separately. Furthermore, we wish to analyze the notion of relative uniqueness, or uniqueness in some respect, doing it at first with the limited resources of predicate logic. There are basically two ways of doing this, depending on the "respect" in which an object is unique. An object may be unique because it is the sole instance of a given property, as is the case with the third power of 2 . Or the object may be unique because of a relation it bears to some other object, as is the case with 
my mother. We shall elucidate these two notions by means of the following definitions.

DEFINITION 1. $a$ is unique in the respect denoted by $F$ iff $a$ exemplifies $F$ and there are no further individuals $x$, other than $a$, that exemplify $F$ :

$$
a \text { is } F \text {-unique }=\text { df } F a \& \neg(E x)(x \neq a \& F x)
$$

or, equivalently,

$$
a \text { is } F \text {-unique }=\text { df } F a \&(x)(F x \rightarrow x=a)
$$

Next we stipulate that " $a$ is $F$-unique" amounts to "the $x$ such that $x$ is an $F^{\prime \prime}$. More explicitly, we lay down

DEFINITION 2. $a$ is (equal to) the $x$ such that $x$ is an $F$, just in case $a$ is $F$-unique:

$$
(a=(\unlhd x) F x)=\operatorname{df} a \text { is } F \text {-unique. }
$$

We have chosen ' $]$ ' to designate the definite descriptor both for typographic convenience and to forestall confusions with Russell's symbol, which designates a different concept.

The above definitions entail our elementary explication of the definite description:

$$
(a=(\square x) F x)=\text { df } F a \&(x)(F x \rightarrow x=a)
$$

For example, "2 2 is the smallest prime", i.e. " $2=(-1) S P x$ ", is now analyzed as: "SP $2 \&(x)(S P x \rightarrow x=2)$ ", where in turn $S P x$ is defined as $(y)(P x \& P y \&$ $x \neq y \rightarrow x<y)$.

For (N) to hold $F a$ need not be true. And if $F a$ is not asserted (separately) then (Ex) $F x$ does not follow. Consequently (N) does not involve or entail existence. For example, the equality

$$
\text { Zeus }=\text { The boss of the Greek Olympus, }
$$

which is a substitution instance of the left hand side of $(\mathrm{N})$, dose not commit us to paganism. It is just a designation convention. If challenged, then the corresponding right hand side will be challenged as well but the equality 
by definition will still hold. Likewise if $(\mathrm{N})$ is construed as an equivalence: for "A↔B" to hold both sides must have the same truth value, e.g. falsity. If we now assert (separately) that Zeus is a boss of the Greek Olympus then we conclude that Zeus exists; and if we deny the same statement, i.e. negate $F a$, then we commit ourselves to the statement that Zeus does not exist.

So much for definite descriptions in terms of unary predicates. We now generalize our analysis to predicates of any rank. But to keep the exposition simple we limit our definitions to binary relations.

DEFINITION 3. $a$ is unique in its relation $R$ to $x$ iff $a$ bears the relation $R$ to $x$ and there are no other individuals $y$, except $a$ itself, that are $R$-related to $x$ :

$a$ is $R$-unique in its relation to $x=$ df $\operatorname{Rax} \&(y)(R y x \rightarrow y=a)$

DEFINITION 4. $a$ is (equal to) the $x$ such that $x$ bears the relation $R$ to $b$, just in case $a$ is unique in its $R$ relation to $b$ :

$(a=(\square x) R x b)=\mathrm{df} a$ is $R$-unique in its relation to $b=\mathrm{df} R a b \&(x)$ $(R x b \rightarrow x=a)$.

To sum up, we have identified definite description with uniqueness. Unlike the accepted view, ours does not involve the assumption that the described individual exists in some context: it only asserts the nonexistence of other individuals satisfying the same conditions. Which is as it should be, for existence is not a matter of definition, let alone of designation: existence is a matter of assumption or of validation. In other words, an existence claim is a hypothesis that must be substantiated, not a convention that can be freely introduced. For example, whether or not a function with given properties exists, is a matter for an existence theorem to decide. And whether or not a concrete object with assumed properties exist, is a matter for empirical tests to decide. These methodological requirements are violated by the construals of Russell and Hilbert-Bernays (see Section 
1) but not by ours. Indeed, on our construal the existence of the described individual may, but need not, be asserted separately, namely thus: $(E x)$ ( $x$ is $F$-unique) or thus: ( $E x$ ) ( $x$ is unique in its $R$ relation to $b$ ). Consequently there will be no logical differences between a proper (or full) description and an improper (or empty) description: the differences are solely semantical. The semantical peculiarities of definite descriptions will be taken up in Sections 5 and 6, in the light of a deeper yet simpler analysis of our subject, to which we turn presently.

\section{A mathematical analysis of definite descriptions}

We shall now avail ourselves of the general concept of a function, which goes beyond the predicate calculus. Consider the formula "The weight of $x$ equals $y$ " or, more briefly, " $x$ weighs $y$ ". Since for every substitution instance of $x$ there is exactly one value of $y$ such that y equals the weight of $x$, weight is a function, so that we can write: " $W(x)=y$ ". Likewise, "The father of $x$ is $y$ ", or shorter " $y$ fathered $x$ ", can be symbolized as: " $F(x)=y$ ", where ' $F$ ' stands for the fatherhood function. These symbols convey the idea that weight and fatherhood are properties of something and moreover that these properties are adequately represented by functions in the mathematical, not in the logical sense, for their values are not statments but further individuals. These examples may also be construed as relational statements with the qualification that the relations $W$ and $F$ be many-one. But this construal, while suitable for ordinary language cases such as "Scott wrote Waverley", is inadequate for most scientific purposes. In science one prefers functional statements of the form " $F(x)=y "$.

Let us now truncate the functional formula "The $F$ of $x$ equals $y$ " by leaving out the value of the function. We thus get the functional semistatement "The $F$ of $x$ " or, shorter, " $F(x)$ ". This expression indicates the func- 
tion and an arbitrary value of its argument but not the value the function takes at that point. If the function does have a value at $x$, i.e. if $F$ is defined at $x$, then that value is unique by definition of a function. And this is all a definite description indicates, namely a unique object.

We compress the above in the following

DEFINITION 5 . Let $F$ be a function from a set $C$ into a set $D$. Then the expression ' $F(a)$ ' is called a proper definite description iff $F$ is defined at $a \in C$.

If $F(a)$ is a proper definite description then it names a unique individual, say $b$, in the codomain $D$. That is, the relation between a proper name $b$ and a definite description $F(a)$ is now the full fledged statement

$$
b=F(a)
$$

read ' $b$ is the $F$ of $a$ '. In this case $F(a)$ is a name and it poses no further problems. Otherwise we have an improper or empty definite description. Moreexplicitly, we lay down

DEFINITION 6. The functional semistatement $F(a)$ is an improper definite description just in case the function $\mathrm{F}$ is not defined at a e $\mathrm{C}$.

Examples: "The weight of my thoughts", "The father of the universe". Suggestion: a number of metaphors are just improper descriptions. This remark might be useful for analyzing the structure of metaphors.

An alternative but essentially equivalent explication is obtained with the help of the concept of a partial function, or a mapping from a subset of $C$ to $D$. Thus "king" and "president" are partial functions on the set of countries: "king" is a total function on the set of monarchies and "president" a total function on the set of republics. In general, we have

DEFINITION 7. Let $F$ be a partial function with domain $C$. Then $F(a)$ is a proper (improper) definite description iff $a$ belongs (does not belong) to $C$. 
A New Look at Definite Descriptions

On either of the two functional construals, the description can be stated and analyzed without even having to introduce a special symbol The link between the preceding elementary elucidation (Section 2 ) and the present one is supplied by the following convention.

DEFINITION 8. Let $P$ be a unary predicate with domain $D$ and let $F$ be a function from a set $C$ into the set $D$. Then

$$
b=(\lrcorner x) P x \text { iff } F(a)=b .
$$

Given a unary predicate $P$ it is always possible to find the corresponding function $F$ that will satisfy the preceding definition, and conversely. For instance, "golden mountain" may be construed not only as a (molecular) predicate but also as a function on the set of places. Consequently, assuming that the name of the golden mountain is 'Shiny', Definition 8 gives us

(Shiny $=$ The golden mountain) iff (The golden mountain at place $a=$ Shiny).

There is more to the relation between predicates and functions.

\section{Continuation of the analysis}

Take "talented" as an example of a unary predicate $P$ and "author of" as an instance of a function $F$. (We brush aside the case of multiple authorship: we restrict our treatment to single valued functions, i.e functions proper.) Both $P$ and $F$ are mappings: $P$ maps writers into statements while $F$ maps books into writers. That is,

$$
\begin{array}{ll}
P & \text { Talented: Writers } \longrightarrow \text { Statements } \\
F & \text { Author of: Books } \longrightarrow \text { Writers }
\end{array}
$$

In the first case we have, e.g., "Walter Scott is talented", while in the second we may have "The author of Waverley is (equal to) Walter Scott". (The is in the first statement is predicative while the is in the second statement is the one of equality.) This allows us to write 


$$
A(w)=s,
$$

where ' $A(w)$ ' stands for "the author of Waverley" and 's' for Walter Scott. By virtue of this, the statement "Walter Scott is talented" can be transformed into "The author of Waverley is talented", i.e. briefly $T(A(w))$. Now, this is just an instance of the composition of the functions $A$ and $T$, i.e.

$$
\text { Books } \stackrel{\text { Author of }}{\longrightarrow} \text { Writers } \stackrel{\text { Talented }}{\longrightarrow} \text { Statements. }
$$

This composition may be represented as a commutative diagram:

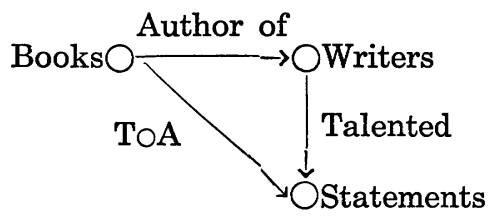

Obviously, this works only when the functions concerned are defined at all the points of the sets involved and when the range of the first is the domain of the second. This remark will help us solve a problem concerning the relation between definite descriptions and names.

As Russell pointed out definite descriptions are not names. They may have the same referent but they do not mean the same. Thus 'The mother of God' "says" more than 'Mary' in the context of Catholic theology. Whatever sense a name may be assigned (and this is a controversial point), the associated definite description, if any, must have a sense containing the previous one. However, proper definite descriptions can be treated, for purposes of deducation, just like proper names. In other words, although a definite description is not a name, it behaves in inference as if it were a name provided the necessary precaution is taken. The condition for this functional or behavioral identity of names and definite descriptions is, of course, that the function $F$ in question be defined at the point concerned. For only in this case will $F(a)$ constitute a proper or nonempty definite 
description. In other words, a definite description can be treated as a name provided there exists an individual complying with the description. Example:

$$
\begin{array}{cl}
T s & 1 \\
A(w)=s & 2 \\
\therefore T(A(w)) & 1,2 \text {, Principle of identity. }
\end{array}
$$

This inference is valid because, on the interpretation given above, the function $A$ is defined at $w$ : indeed, at $w$ A takes on the value $s$. On the other hand, if now ' $w$ ' stands for the world, then in the context of a naturalistic world view the above inference is invalid no matter how ' $T$ ' be reinterpreted, for $A$ is no longer defined at $w$. In short, with this new interpretation of ' $w$ ', $A(w)$ becomes an improper description and the substitution of $A(w)$ for $\mathrm{s}$ is invalid because one of the conditions for the composition of functions, i.e. for the existence of the composite $T o A$, is no longer met. We may, if we wish, say with Frege that ' $A(w)^{\prime}$ ' now designates the null individual, but this won't save the inference. In this regard, then, our treatment does not differ from either Russell's or Hilbert-Bernays'. The difference is, of course, that the existence condition is now stated separately instead of being fused with the definite description.

\section{Meaning}

Meaning is contextual: 'The creator of the universe' makes sense in theodicy but not in physics, where the very concept of creator does not occur. Hence we had better start by giving a quick elucidation of the notion of a context.

DEFINITION 9. The ordered triple $C=\langle U, F, S\rangle$ is called a context iff $S$ is a set of statements in which only the function constants in the function family $F$ occur, and the reference class of every $F$ in $F$ is included in the universe or domain of individuals $U$. 
We now postulate the conditions under which a definite description, construed as a functional semistatement (Section 3), makes sense in a given context and has a referent in it.

AXIOM 1. The definite description $F(x)$ makes sense in the context $C=\langle U, F, S\rangle$ iff $F$ is in $F$

AXIOM 2. The definite description $F(x)$ has a referent in the context $C=\langle U, F, S\rangle$ iff $F(x)$ makes sense in $C$ and $F$ is defined at $\mathrm{x}$ e $U$.

DEFINITION 10. Let $F(x)$ be a definite description that makes sense and has a referent in a context $C=\langle U, F, S\rangle$. Then the value $y=F(x)$ is called the referent of $F(x)$.

(Notice that the above axioms are postulates indeed and not definitions, for they do not define sense and reference: they only stipulate necessary and sufficient conditions for a description to have both sense and reference.)

Now, in our view, for an expression to be meaningful, it is necessary for it to have a sense. It follows that 'The present king of France' and other referentially vacuous definite descriptions may be meaningful in certain contexts. In other words, an empty description may stand for a concept, i.e. something endowed with a sense or intension, even though it fails to denote, i.e. to have a real counterpart.

This view is at variance with Austin's ${ }^{4}$ that empty descriptions and sentences containing them "suffer from infelicity" because devoid of real reference. This doctrine would be justified if meaning were identified with reference or, even more drastically, with extension (adequate reference). But such a referential view of meaning would be crippling to science, where statementes about "theoretical entities" have to be made, discussed and put to the test long before one can ascertain whether they have real referents. 


\section{Truth}

Consider the much discussed statement "The king of France is bald". Since it contains an empty description, can we say that it is true or that it is false? Russell held it to be false and most philosophers seem to share this view for the simple (and therefore suspect) reason that statements of that kind are clearly not true and, since they are propositions and propositions are allegedly, by definition, either true or false, then they must be false. However, some philsophers have felt dissatisfied with this view. Thus at one time Strawson ${ }^{5}$ endorsed Frege's view that statements containing empty descriptions are neither true nor false-a view Quine ${ }^{6}$ baptized the truth value gap theory. More recently Strawson ${ }^{7}$ has come to the conclusion that his former view is not compelling: that each side has its merites and it is unimportant which is taken. This may well be so in the soft open context of descriptive semantics. In a theory proper any statement concerning the truth value of a proposition containing an improper description should be decidable.

In our view truth and falsity do not inhere in propositions but are attributed to them: i.e. truth values are values of a partial valuation function $V$ defined on the set of propositions. Now, in order for a statement containing a definite description to be assigned a truth value in a given context, it must point to a referent in that context. For, if it fails to have a referent, then it cannot be "faced" to its referent in order to assign it a truth value. (And if the statement does have a referent then it also has a sense by Axiom 2 in Section 5.) Therefore we stipulate

AXIOM 3. A statement containing a definite description $F(x)$ may be assigned a truth value in the context $C=\langle U, F, S\rangle$ iff $F(x)$ has a referent in $C$.

For example, "the luminiferous ether" may be regarded as a definite 
description the sense of which is determined by the axioms of some ether theory. In all mechanical ether theories, like Cauchy's, "The luminiferous ether is elastic" is taken to be true. But the same statement makes no sense in contemporary optics, hence it can be assigned no truth value in this context. In other words, the predicate "ether" does not belong to the function family in the context of modern optics. Hence the valuation function $V$, that carries the couples statement-context into truth values, is not defined for the pair (The luminiferous ether is elastic, Modern Optics). In obvious symbols, while $V(e, E)=1, V$ takes no value at $(e, M O)$, i.e. $e$ has no truth value in or relative to $M O$. (Nor does $e$ have the "indeterminate" value, as some interpretations of many valued logic would have it. A function not determined, or not defined, for a certain value of its argument $h$ as no value at it. Depending on the theory of truth, $V$ may take two or more values but it cannot have the value "indeterminate".)

\section{The real size of the theory of descriptions}

The analysis of definite descriptions has been overrated by some logicians (for example Hilbert and Bernays devote it 70 pages). It has even been regarded as Russell's main contribution to philosophy-which is a sly way of belittling $P M$. On the other hand the theory of descriptions has been underrated and even misplaced by some ordinary language philosophers. Some took it to be a matter for literary criticism, others for a grammatical analysis of the definite article-as if 'my wife,' 'Plato's teacher', and 'that weird chap in the corner' did not qualify as definite descriptions.

There is little doubt that all advanced ordinary languages teem with definite descriptions even if they lack definite articles. Mathematics-the language of science-is full with definite descriptions too-just recall "the sine of $10^{\circ}$, 'the composition of $f$ and $g$ ', and 'the indefinite integral of $f^{\prime}$. 
If so it behoves the philosopher to analyze such an ubiquitous concept. But the analysis need not introduce any new technical concept: we have seen that descriptors are reducible to standard components of logic and mathematics. Our view of the theory of descriptions lies thus mid-way between the two extreme theories that are currently dominant: instead of either renouncing or inflating definite descriptions, we hold them to be normal constituents of any language with a reasonable expressive power. The syntax of definite descriptions is trival: only their semantics is somewhat sophisticated.

I wish to record my indebtedness to my colleague J. Lambek, my associates F. Oppacher and $\mathrm{H}$. Kurosaki, and my student C. Castonguay for their criticisms of a draft of this paper.

\section{REFERENCES}

1. B. Russell, "On denoting", Mind, N.S., 14, 479 (1905). A. N. Whitehead and B. Russell, Principia Mathematica, vol. 1 (Cambridge: Cambridge University Press, 1910), Introduction, Ch. III and *14. B. Russell, Introduction to Mathematical Philosophy (London: Allen and Unwin, 1919), Ch. XVI.

2. D. Hilbert and P. Bernays, Grundlagen der Mathematik, vol. 1 (Berlin: SpringerVerlag, 1934), Ch. 8.

3. D. Kalish and R. M. Montague, "Remarks on descriptions and natural deduction", Archiv f. Math. Logic und Grundlagenforschung 3, 50 (1957), particularly p. 59. D. Scott, "Existence and description in formal logic", in R. Schoenman, Ed., Philosopher of the Century: Essays in Honour of Bertrand Russell (London 1967).

4. J. L. Austin, How To Dog Things with Words (Cambridge, mass: Harvard University Press, 1962).

5. P. F. Strawson, "On referring", Mind, N.S. 59, 320 (1950).

6. W. v. O. Quine, Word and Object (Cambridge, Mass.: M.I.T. Press, 1960).

7. P.F. Strawson, "Identifying reference and truth-values", Theoria, 30, 96 (1964). 
マリオ・ブンゲ, Mario Bunge

1919年, アルゼンチン; ブェノスアイレスで生れる. 1952年, 23歳で, アルゼンチン， ラプラタ大学より $\mathrm{PhD}$ (理論物理学)を受け, ブエノスアイレス, ラプラタ, テンプル, デ ラウエア各大学教授(理論物理学)経て, 現在, カナダ, マクギル大学教授(哲学)兼同大学 Foundations and Philosodhy of Science Unit 所長, 著書K, Causality, Metascientific Queries, Cinemática del electrón relativista, Intuition and Science, The Myth of Simplicity, Foundations of Physics, Scientific Research があり, との 他物理学及び哲学に関して, 100 篇以上の論文がある. 更に編著には, Studies in the Foundations, Methodology and Philosophy of Science Vol. I,II, the Critical Approach to Science and Philosophy, Library of Exact Philosophy があって, ての 方面の活躍も著しけ: 